Historic, Archive Document

Do not assume content reflects current scientific knowledge, policies, or practices. 

f

(t)

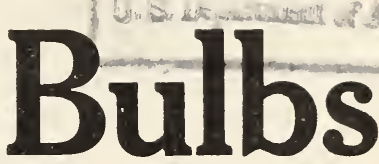

\section{WHOLESALE AND RETAIL PRICE LIST}

JANUARY 1928

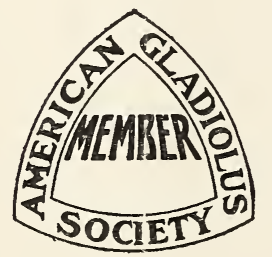

\section{LEGron Floral Co.}

\section{Amherst Drive}




\section{LeGRON FLORAL CO., 125 Amherst Drive, Toledo, Ohio}

\section{WHOLESALE PRICE LIST-JANUARY, 1928}

All wholesale prices are F. O. B. Toledo, Ohio.

Twenty-five per cent will hold order for spring delivery.

Bulbs will be sent C. O. D. at any time with an advance payment of $25 \%$.

All orders accepted subject to prior sales.

As season advances we will be out of certain sizes. Shall we substitute the next size if we are out of one ordered?
We do not substitute varieties unless requested to do so. Make your wants clearly known in first order as frequently while we are corresponding regarding a certain size some other customer sends a check in for the whole lot.

We sell at wholesale to any one providing you take in quantities as listed.

Minimum sales are $\$ 1.00$ for your total items.

Special prices given to Jobbers ordering in lots of 10,000 or more.

\section{VARIETY}

Foch, Le Marechal

Halley

Herada

King, Mrs. F.

Kirtland, Evelyn

Mixed

Prim Hyb. Mixed
Per 1000 (250 at 1000 rate)

No. 1 No. 2 No. 3

$1 \frac{1}{2}$ Up $1 \frac{1}{4}-1 \frac{1}{2} \quad 1-11 / 4$

$\$ 12.00$

16.00

18.00

25.00

15.00

15.00

$\$ 10.00$

13.00

20.00

14.00

20.00

12.00

12.00
No. 5

$1 / 2-3 / 4$

$\$ 3.50$

3.00

8.00

4.00

5.00

16.00

10.00

10.00
Albania

Alshira

Arden

Ardmore

Armsby, Mrs. J. K.

Baker, Capt. A. C.

Baltimore

Bertrex

Bobolink

Bothin, Mrs. H. E.

Charlemagne

Coleman, Joe

Coulter, John Merle

Crescent Queen

Crimson Glow

Elf

Elora

Empress of India.

Fairfax

Fennell, Mary

Foch, Le Marechal

Franklin, Helen

Giant Nymph

Glory of Holland

Golden King

Golden Measure

Halley

Herada

Hulot, Baron Joseph.

Jewell

Kent, Mrs. Wm.

King, Mrs. F.

Kirtland, Evelyn

Kunderdii Glory

Lehman Lily

Lily White

\section{$\$ . .4 .00$}

4.00

4.00

4.00

12.00

...........

2.50

4.00

5.00

4.00

.........

….......

…... 4.00

4.00

5.00

3.00

1.50

2.50

12.00

...........

6.00

1.80

3.00

4.00

4.00

4.00

2.00

3.00

6.00

4.00

2.50

3.25
3.25
3.25
3.25
$\ldots . .$.
3.25
2.50
2.00
3.25
4.00
3.25

3.25

3.25

3.25

4.00

2.50

1.20

2.00

10.00

2.00

3.25

5.00

1.50

2.50

3.25

3.25

3.25

1.75

2.50

5.00

3.50

2.00

\section{Per 100 (25 at 100 rate)}

…... 3.25

…... $\quad 2.00$

$4.00 \quad 3.25$

2.50

2.50

2.50

8.00

2.50

2.00

1.75

2.50

3.25

2.50

3.25

2.00
3.25

3.00

2.50

2.50

3.25

2.00

1.00

1.75

8.00

1.50

2.50

4.00

1.25

2.00

2.50

2.50

2.50

1.50

2.00

4.00

3.00

1.50
1.50

Bulblets in quanti-

No. 6 ties as listed only $1 / 4-1 / 2$

$\$ 2.00$

2.00

4.00

2.00

3.00

.........

Qt.

Bu.

$\$ .50 \quad \$ 8.00$

.75

1.50

.75

1.00

.50

.50

8.00

10.00

20.00

8.00

8.00

Bulblets

$100 \quad 1,000$

1.00

1.50

1.50

1.50

4.00

1.50

1.25

1.00

1.50

2.00

1.50

2.00

2.00

1.50

2.00

2.00

1.50

1.50

2.00

1.50

$\begin{array}{r}.75 \\ \hline\end{array}$

1.25

5.00

1.00

1.50

2.75

.75

1.25

1.25

1.25

.75

1.00

2.50

2.50

\subsection{0}

1.00

1.00

2.50

1.00

.75

.75

1.00

1.50

1.00

1.50

1.50

1.00

1.50

1.50

1.00

1.00

1.50

1.00

.50

1.00

3.50

1.00

2.00

.50

1.00

1.00

1.00

.50

.75

2.00

.50 


\begin{tabular}{|c|c|c|c|c|c|c|c|}
\hline \multirow{3}{*}{ VARIETY } & \multirow[b]{3}{*}{$\begin{array}{c}\text { No. } 1 \\
11 / 2 \text { Up }\end{array}$} & \multirow{2}{*}{\multicolumn{4}{|c|}{ Per 100 (25 at 100 rate) }} & \multicolumn{2}{|c|}{ Bulblets } \\
\hline & & & & & & 100 & 1,000 \\
\hline & & $\begin{array}{l}\text { No. } 2 \\
11 / 4-11 / 2\end{array}$ & $\begin{array}{r}\text { No. } 3 \\
1-11 / 4\end{array}$ & $\begin{array}{l}\text { No. } 5 \\
1 / 2-3 / 4\end{array}$ & $\begin{array}{c}\text { No. } 6 \\
1 / 4-1 / 2\end{array}$ & & \\
\hline London, Jack & & 5.00 & 4.00 & 2.50 & 2.00 & & \\
\hline Maiden's Blush & 2.50 & 2.00 & 1.50 & ......... & ......... & $\cdots$ & ….... \\
\hline Majestic & .......... & 2.50 & 2.00 & 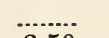 & ........ & & \\
\hline Ming Toy & & 5.00 & 4.00 & 2.50 & & .25 & 2.00 \\
\hline Muriel & 5.00 & 4.00 & & 2.00 & 1.50 & & \\
\hline 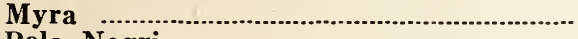 & 3.00 & 2.50 & 2.00 & 1.25 & .75 & .25 & 2.00 \\
\hline Pola Negri ... & ......... & & & 2.00 & 1.50 & .20 & ........ \\
\hline Niagara & ......... & 2.50 & 2.00 & 1.25 & 1.00 & ......... & ........ \\
\hline 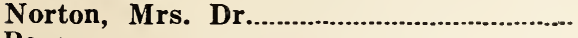 & & 3.00 & 2.50 & 1.75 & 1.25 & ......... & ........ \\
\hline Peace & 2.50 & 2.00 & 1.60 & .75 & .50 & ......... & ....... \\
\hline Pendleton, Mrs. Frank.................................... & 2.50 & 2.00 & 1.60 & .75 & .50 & .......... & \\
\hline Pickford, Mary & & 4.00 & 3.25 & 2.00 & 1.50 & & 1.00 \\
\hline Pink Wonder & 6.00 & 5.00 & 4.00 & 2.50 & 2.00 & .35 & ......... \\
\hline 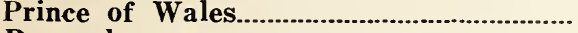 & & 3.00 & 2.50 & 1.75 & 1.25 & .25 & \\
\hline Remembrance & 6.00 & 5.00 & 4.00 & 2.00 & 1.50 & .25 & 1.50 \\
\hline 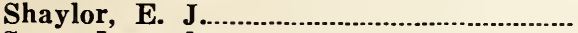 & 4.00 & 3.25 & 2.50 & 1.75 & 1.25 & .25 & 1.00 \\
\hline Sweet Lavender & 5.00 & 4.00 & 3.25 & 2.00 & 1.50 & .25 & 2.00 \\
\hline Sweet Orra & 1.50 & 1.25 & 1.00 & .50 & & ........ & .50 \\
\hline Tiplady, Alice & 2.50 & 2.00 & 1.60 & .75 & .50 & ......... & 1.00 \\
\hline 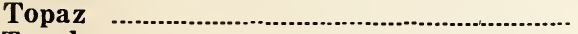 & 4.00 & 3.25 & 2.50 & 1.75 & 1.25 & ......... & 1.00 \\
\hline 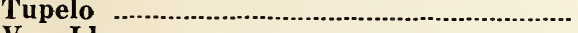 & 4.00 & 3.25 & 2.50 & 1.75 & 1.25 & ........ & 1.00 \\
\hline Van Ida & 2.00 & 1.60 & 1.30 & ......... & -........ & ........ & -....... \\
\hline Watt, Mrs. & 3.00 & 2.50 & 2.00 & ......... & ........ & .......... & -....... \\
\hline 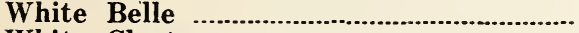 & 4.00 & 3.25 & & ......... & ......... & ......... & ....... \\
\hline White Cluster & 4.00 & 3.25 & 2.50 & & & & 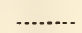 \\
\hline White Wonder & 5.00 & 4.00 & 3.25 & 2.00 & 1.50 & .35 & ....... \\
\hline Wilbrinck & 2.50 & 2.00 & 1.50 & 1.00 & .75 & ........ & .75 \\
\hline 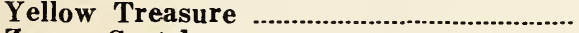 & & 5.00 & 4.00 & 2.50 & 2.00 & ......... & 1.00 \\
\hline Zang, Gretchen & 2.00 & 1.50 & 1.25 & .75 & .50 & ......... & .35 \\
\hline 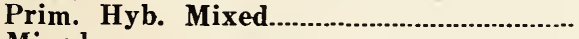 & 1.50 & 1.20 & 1.00 & ........ & ........ & ......... & ......... \\
\hline Mixed & 1.50 & 1.20 & 1.00 & ...... & ...... & ..... & ........ \\
\hline
\end{tabular}




\section{LeGRON FLORAL CO., 125 Amherst Drive, Toledo, Ohio}

\section{RETAIL PRICE LIST-JANUARY 1928}

We will issue no catalog this season. We have had a wonderful growing season and ideal weather for digging so that we have about three times as many bulbs this year per dollar expense as we had last. Retail prices have been cut accordingly from $25 \%$ to $70 \%$ less than last year. We have 146 varities not listed on this list that we are going to put on a CLOSE OUT LIST as soon as bulbs are cleaned, graded and counted. Our stock has increased to such an extent that we will simply have to let some of our varieties go entirely. Be sure and get this CLOSE OUT LIST. Ready about December 10th, 1927.

Varieties on this list will not be on the CLOSE OUT LIST so order in time if you wish any of the varieties listed here at prices quoted. Nearly all bulbs sold last season at $15 \mathrm{c}$ to $45 \mathrm{c}$ have been reduced this year to $10 \mathrm{c}$.

TERMS: Cash with order or $25 \%$ deposit and balance when you are ready for the bulbs. Bulbs will be sent C. O. D. if $25 \%$ deposit is advanced.

\section{VARIETIES}

Albania, white.

Alma, light pink

Alshira, dark wine red.

Altair, salmon.

Altamyra, lilac pink.

Anthem, light rose red.

Arbutus, arbutus pink

Arcola, red, very late.

Arden, watermelon red.

Ardmore, lavender pink

Armour, J. Ogden, lavender rose.

Armsby, Mrs. J. K., coral pink.

Ashe, Emile, white.

Atlantis, geranium pink.

Atreus, rose pink

Autumn, late red

Baker, Capt. A. C., dark red

Baltimore, salmon pink.

Beacon Flame, red.

Beale, Mrs. Truxton, cream

Beatrice, pink and cream

Belvedere, light salmon.

Bengal Tiger, mottled red

Bennett, Dr. F. E., peach red.

Bertrex, white.

Blue Isie, lilac blue.

Blue Jay, light blue.

Blue Ribbon, dark lilac blue.

Bobby, dark rose.

Bobolink, orange pink.

Bolinas, cream white.

Bollinger, Blanche, pink

Bothin, Genevieve, smoky pink

Bothin, Mrs. H. E., light pink

Boynton, Capt., lavender.
POSTAGE AND EXPRESS. All bulbs in the retail list are sent out by us prepaid, but bulbs from wholesale list are F. O. B. Toledo, Ohio. If you mix your orders send enough extra to cover transportation on bulbs ordered wholesale.

We will refund any excess you send. Do not write asking us what it will be as we can not tell until order is packed. After order is packed it should be sent at once and not held for correspondence. Bulbs packed ready for shipment in large size (Nos. 1 and 2 combined) will weigh about six pounds to the 100 .

Your Postmaster or Express Agent can give you the rate from Toledo, Ohio.

Postage collected C. O. D. costs the customer about $15 \mathrm{c}$ extra.

We ship bulbs all winter at our own risk. Valuable packages should be sent express in cold weather as $\mathrm{P}$. O. does not insure against freezing.

Prices per single bulb

One dozen for price of ten

in same variety and size

Large Medium Small

$1 \frac{1}{4}$ Up $3 / 4-1 \frac{1}{4} \quad 1 / 4-3 / 4$

$\$ .10$

1.00

$\$ .07$

.10

.10

.15

.35

.15

.10

.10

1.00

.15

.15

.20

.10

.15

.10

.10

.10

.10

.25

1.00

.10

.50

.05

.15

.10

.30

1.00

.15

.50

1.00

.30

.10

.20
$\$ .05$

.75

.07

.07

.25

.10

.25

.10

.07

.07

.10

Dozen $\begin{array}{cc}\text { Bulblets per } \\ 100\end{array}$

.50

.05

.05

.15

.15

.05

.05

.07

.10

.15

.07

.10

.07

.07

.07

.07

...........

.35

.10

.07

.60

.10

.35

.20

.07

.15

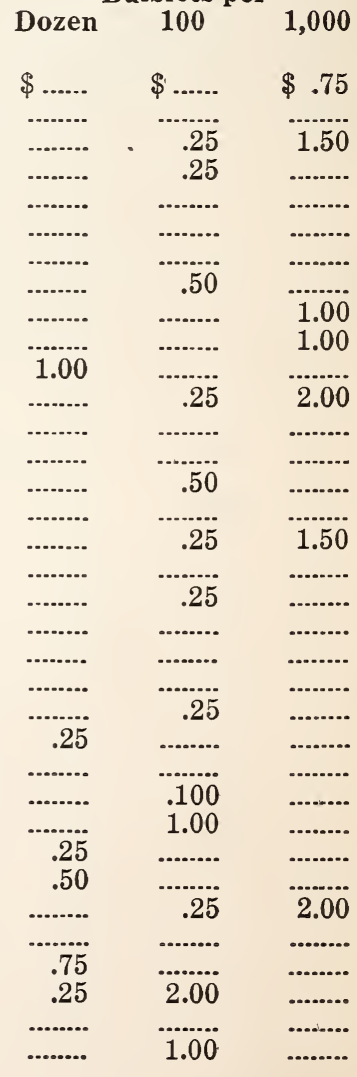




\section{VARIETIES}

Bubbles, shrimp pink

Bumblebee, salmon rose pink

Burke, Mary Stearns, yellow.

Californita Giant, light purple

Carbone, J. A., red

Cardinal Prince, red

Carmen, Sylvia, white

Caruso, red

Cattlaya, pink and cream

Challenger, red

Chalice Flower, white

Charlemagne, red.

Cherry King, red.

Coleman, Catherine, pink

Coleman, Joe, red

Conspicuous, blue

Cooper, Madison, maroon

Cothran, Dr. Lincoln, pink and steel blue.

Coulter, John Merle, dark red.

Crawford, Matthew, creamy pink

Crescent Queen, dark wine.

Crimson Glow, red.

Crinkles, peach blossom pink

Cruentes Superba, scarlet.

Cunningham, Mrs. Robt., pink, lilac and ruby....... 1.00

Defiance, blush-white...

Del Monte, aster purple.

Del Oro, salmon.

Deiner's American Beauty

Deiner, Richard, pink

Dixie, black-red.

Doro, cherry-red.

Douglass, Mrs. Leon, begonia rose

Draco Gloriosa, buff

Dream, salmon red

Dunlaps, red

Early Black, dark red

Eberius, Anna, rhodamine purple

Ebony, dark red.

Edison, Thomas A., begonia rose

Eldiva, cerise.

Elena, white with blotch

Elf, creamv white.

Elkhart, violet purple.

Elkins, Dr., white with purple blotch

Elora, white with yellow blotch.

Empress of India, maroon.

Esberg, Caroline, flame scarlet.

Ethelyn, orange yellow

Evening Glory, rose pink

Ewbank, Rev., porcelain blue

Exquisite, American Beauty.

Fairbanks, Chas. F., red

Fairfax, magenta

Farrar, Geraldine, blue

Fay, Miss Maud, Amaranth pink

Fennell, Mary, deep lilac

Fernekes, Martha, deep blue

Field, Joseph, dawn pink

Fisher, Virginia, rose pink.

Foch, Le Marechal, light pink.

Foch, Marshall, rose pink.

Fontaine, peaches and cream.
Prices per single bulb

One dozen for price of ten

in same variety and size

Large Medium Small

$11 / 4$ Up $3 / 4-11 / 4 \quad 1 / 4-3 / 4$

\section{.15}

.15

.10

.75

.15

1.25

.10

.40

.75

.10

1.00

.10

.10

.50

.10

.10

.10

.15

.10

.15

.10

.10

.25

.10

.25

.40

.15

.20

.10

.75

.15

.15

.15

.15

.10

.10

.15

.50

.40

.50

.10

.20

.20

.10

.10

.25

.75

.10

.40

.10

2.00

.10

.10

.40

1.00

.35

.05

.10

.30

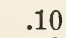

.10

.07

.10

.75

.07

.30

.50

.07

.07

.........

.07

.07

.10

.07

.10

.07

.07

.20

.25

.75

.07

.20

.30

.10

.15

.07

.60

.10

.10

.10

.10

.07

.07

.10

.40

.30

.35

.07

.15

.07

.07

.50

07

.07

.30

.07

1.50

.07

.07

$-.25$

.75

.20

.07

.20
.07

.05

.25

.07

.50

.05

.20

.25

.05

.05

.05

.25

.05

.05

.07

.05

.07

.05

.05

.15

.50

.05

.10

.20

.07

.10

.05

.40

.07

.07

.07

.07

.05

.05

.07

.25

.20

.05

.07

.05

.05

.25

.05

.07

.20

.05

.05

.05

.50

.........

.05

.10
Dozen $\begin{gathered}\text { Bulblets per } \\ 100\end{gathered}$

1,000

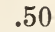

.75

1.75

.50

1.00

1.00

.15

.50

.15

.25

........
......
.35

.......... 


\section{VARIETIES}

Ford, Henry, dark Rhodamine purple.

Franklir, Helen, white

Fryer, W. E., deep violet.

Gallant Leader, see special description

Gaynore, begonia rose.

Geisha, cream yellow.

Gerberding, Elizabeth, shell pink

Giant Nymph, rose and cream.

Glendale, American Beauty

Glory of Holland, white.

Goehl, Henry C., white and red blotch.

Gold, yellow.

Gold Eagle, yellow

Golden Glory, yellow.

Golden King, yellow

Golden Measure, yellow.

Golden Salmon, salmon

Golden Swallow, yellow.

Halley, salmon pink

Hardee, Elizabeth, pink

Hawaii, rose pink

Heart of Juanita, cherry

Heavenly Blue, blue.

Helga, salmon pink

Herada, mauve.

Herbstzauber, red

Hess, Katherine F., deep pink.

Highland Laddie, pink

High Nye, old rose.

Hulot, Baron Joseph, purple

Ibanez, Blasco, dahlia purple

Illuminator, carmine red.

Imperator, white

Indian, analine red

Indianapolis, violet

Indian Summer, late pink

Insull, Mrs. Samuel, blush white

Jackson, Dr. R. T., deep red.

Jacoba, Van Beiren, violet.

Jap Lady, Bishop purple

Jewell, salmon pink

Joerg's White, white

Jordan, David Starr, flame.

Jumbo, pink

Juno, orange.

Kent, Mrs. Wm., flaked pink

Kent, Thomas T., pink striped

King, Mrs. Francis, red.

King Smoke, smoky blue.

Kirtland, Evelyn, rose pink

Kiva, yellow pink

Konynenburg, Mrs. Van, blue.

Krausgrill, Norma, light rose pink.

Kunderd, Anthony, B., cream and white

Kunderd, Marie, white

Kunderd, Mrs. A. E., white

Kunderd, Robert J., scarlet.

Kunderdii Glory, cream-pink.

Kunderd's No. 163, pink.

Kuroki, General, rosy red

Kyle, Fern, creamy white

Lacinatus, old rose.

La Gaiety, deep pink
Prices per single bulb

One dozen for price of ten

in same variety and size

Large

$11 / 4$ Up

Medium

Small

.25

.10

.10

5.00

.40

1.00

.10

.15

.10

.05

.20

.10

2.50

.10

.10

.10

1.00

.15

.05

.40

.10

.15

7.50

.10

.05

.50

.10

.50

.75

.10

.75

.25

.35

.15

.15

.40

.50

.10

.15

1.00

.10

1.00

.15

.10

.10

.10

.10

.05

.25

.05

.50

4.00

.20

.15

.10

.10

.35

.10

.15

.50

.25

1.50

2.00
$3 / 4-11 / 4$

.15

.07

.07

.30

.75

.07

.10

.07

.10

.07

1.75

.07

.07

.07

.75

.10

.30

.07

.10

.07

.40

.07

.40

.07

.60

..............

.10

.10

.30

.40

.10

.75

.07

.10

.07

.07

.07

.15

.40

3.00

.15

.10

.07

.25

.07

.10

.15

1.25
$1 / 4-3 / 4$

.05

.05

.20

.50

.05

.07

.05

.05

1.25

.05

.05

.05

.50

.07

.20

.05

.07

3.00

.05

.30

.05

.05

.35

..........

.07

.07

.20

.30

.07

.50

.05

.07

.05

.05

.05

.10

.30

2.00

.10

.05

.15

.05

.07

.10

1.00

1.00
Dozen

Bulblets per 100

1,000

.15

.50

1.00

2.00

1.25

….... $\quad . . . . . .5$

.50
.35

4.00

.50

.50

5.00

…..... $\quad . .25$

1.00

.........

........

.......

.50

7.50

.50

.50

.75

.75

.25

.50 
Prices per single bulb

VARIETIES

Lehman, Lily, white

Leota, clear pink

Lily White.

Lily Blotch, pink-red blotch

Lind, Jennie, orange pink

Lohrman, Mrs. Richard, shell pink

London, Jack, salmon with red stripes

London Smoke, smoky blue

Lorely, yellow

Lorice, scarlet.

Los Angeles, shrimp pink

Louise, lavender.

Loveliness, cream

Lucia, Amaranth pink

Lucette, orchid pink

McCutcheon, John T., red

Magic, lavender blue.

Maiden's Blush, light pink

Maine, citron yellow.

Majestic, stone red.

Malvern, rose pink and pansy throat

Mark, Mr., dark blue.

Marnia, orange.

Martz, Dr. Christ, light red

Masterpiece, American Beauty

Master Wietze, red with white lines

Meeker, Mrs. Arthur, American Beauty

Michelena, Beatriz, orange.

Milady, creamy white.

Millionaire, crimson.

Ming Toy, buff and yellow

Minuet, lavender.

Miss Spokane, orange salmon

Mona Lisa, lavender.

Monon, dark rose pink.

Moore, Pres. C. C., begonia rose.

Muriel, blue.

Myra, salmon.

Myrtle, light pink

Nampa, white

Nautilus, shell pink

Negri, Pola, apricot.

Neoga, garnet red:

Niagara, yellow

Nightingale, rose pink

Nineteen-Ten Rose, rose

Norton, Mrs. Dr., pink and cream.

Old Glory, dark red striped white.

Oliver, J. D., lavender rose

Olson, Esther, buff.

Opalescent, rose-lavender

Orange Glory, orange.

Orange Queen, orange.

Orofino, old rose pink

Paramount, pink and cream

Pasadena, Turkish red.

Peace, white

Pendleton, Mrs. F., pink with ruby blotch

Perla, cherry crimson.

Persia, deep maroon.

Peters, Mrs. F. C., lavender

Pfitzer's Triumph, brilliant red

Phaenomen, pink and yellow
One dozen for price of ten

in same variety and size

Large Medium Small

$11 / 4$ Up $3 / 4-11 / 4 \quad 1 / 4-3 / 4$

.10

.10

.10

.15

.10

.15

1.00

.10

.10

.10

.40

.10

1.00

.10

.05

.10

.10

.15

.10

.25

.75

.10

.10

.10

.50

.15

.75

.10

2.50

.15

.10

.10

.10

.10

.10

.10

.10

.20

.10

.10

.05

.10

.10

.10

1.00

.15

.10

.25

.10

.25

.05

.05

.50

15

3.00

.60
.07

.07

.07

.10

.10

.10

.07

.10

.10

.05

.05

.05

.07

.07

.07

.05

.07

.50

.05

.05

.20

.50

.05

.05

.05

.07

.05

.15

.50

.05

.05

.20

.35

$\ldots$

.07

.10

.07

.07

.07

.07

.07

.07

.07

.07

.07

.07

.07

.07

.10

.75

.35

.07

.07

.25

.15

.05

.........

.05

.05

.05

.05

.05

.05

.05

.05

.05

.05

.05

.05

.50

$.20^{\circ}$

.05

.05

.15

.10

.40

.10

.10

.40
Dozen $\begin{gathered}\text { Bulblets per } \\ 100\end{gathered}$

.20

.20

1.00

.50

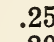

.20

1.00

.20

.25

.75

.25

.50

.20

.75

.25

.15

.15

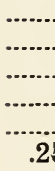

2.00

.25 
Prices per single bulb

\section{VARIETIES}

Phipps, Wm. H., pink

Phini, pink striped purple

Pickford, Mary, white and cream

Pinnacle, salmon.

Pirie, John T., mahogany brown.

Pink Lily, pink.

Pink Perfection, pink.

Pink Wonder, pink

Plath, Gladys, orchid with blotch

Porter, Gene Stratton, soft pink

Princeps, red.

Prestgard, Mrs. Kr., white.

Pride of Goshen, salmon pink.

Primunella, orange-saff ron.

Prince of India, deep smoke.

Prince of Wales, salmon

Princess of Orange, orange.

Purple Glory, deep maroon.

Purple Queen, purple.

Purple Spot, purple.

Queen of the Night, deep maroon

Quinton, coral pink.

Rarity, lavender and old rose.

Ravenna Cream, yellow

Red Amarillas, red.

Red Copper, red

Red Emperor, red.

Red, White and Gold

Remembrance, rose pink

Richardson, Mrs. Willard, crimson

Roem, Von Kennemerland, pink

Romance, old rose.

Rose Ashe, ash rose.

Rose Glory, rose

Rosella, light rose and purple.

Rosemary, white with lavender stipplings.

Rose Precose, rose pink

San Anselmo, white and pink.

San Jose, apricot and red.

Scarlano, red.

Scarlet, scárlet red

Scarlet Princeps, red.

Scarlet Wonder, red

Schwaben, yellow.

Schweppe, Mrs. Laura Shedd, peach pink

Selma, rose and yellow

Sentinel, rose pink

Sharon Rose, rose.

Shaw, Bernard, light pink.

Shaylor, E. J., rose pink

Sheila, salmon.

Shook, Dr. Nelson, rose

Sierra, cream-rose-lilac.

Silver Lace, white

Sisson, Mrs. P. W., pink

Smith, Byron L., lavender pink

Snow Glory, white

Sonoma, canary yellow.

Springer, Ed., rose pink

Stalwart, yellow.

Storm King, red.

Sulphur Frills, yellow

Sulphur Glow, yellow.
One dozen for price of ten

in same variety and size

Large Medium Small

$11 / 4$ Up $3 / 4-11 / 4 \quad 1 / 4-3 / 4$

.50

.25

1.00

.35

.50

.05

.10

.25

.10

.10

.10

.15

.50

.10

.75

.10

.10

.15

.05

.10

.10

.10

.15

.20

1.50

.10

.15

.10

.10

.10

.25

.05

.10

.10

.10

.10

.50

.15

.10

.50

.10

.75

.10

.25

.05

.35

.35

.10

.25

1.00

.10

.10

.75

.20

.15

.10

.50

50

$.50-.20$
$-\quad .25$

.35

.10
.07

.20

.07

.07

.10

.30

.07

.07

.07

.10

.07

1.00

.07

1.00

.10

.15

.07

.10

.07

.07

.20

.07

.07

.07

.07

.40

.10

.07

.40

.07

.60

.07

.20

.25

.25

.07

.20

.75

.07

.07

.60

.15

.10

2.00

.07

.40

.25

.20

.10

.07
.15

.05

.05

.07

.20

.05

.50

.05

.05

...........

.05

.75

.05

.75

.07

.10

.05

..........

.05

.15

.05

.05

.05

.05

.30

.07

.05

.25

.05

.40

.05

.15

.20

.05

.15

.50

.05

.05

.50

.07

1.00

.05

.30

.15

.07

.15

.05
Bulblets per 100

1,000
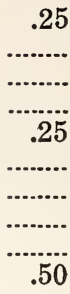

1.50 
Prices per single bulb

VARIETIES

Summit, cherry red

Superba, soft carmine

Surprise, yellow and pink.

Sweet Lavender, lavender.

Sweet Orra, light yellow.

Symmes, Frank J., peach red

Tabor, Elizabeth, pink with red blotch

Taft, Pres., pink

Taylor, Ruth, white

Tempa, lavender pink

Theda, shrimp pink

The President, red

Thompson, Anne, cream and pink

Tiger, salmon red and smoky blue.

Tiger Face, crimson striped.

Tilley, Vesta, rosy white

Tiplady, Alice, orange-saffron.

Todd, Helen, rose pink.

Topaz, pink and buff.

Trousseau Pink, see special description.

Tupelo, yellow and red.

Uhlan Chief, scarlet

Valdemar, Thais, old rose

Van, Ida, cherry red.

Vanderbilt, Mrs. Newell, salmon pink

Van Fleet, Dr., pink and cream

Vanity, LaFrance pink

Veneta, deep wine.

Victor, yellow.

Vielchenblau, blue.

Violet Beauty, cerise violet

Violet Glory, deep violet.

Virginia, rose pink.

Waikiki, flesh pink.

Walsh, Mrs. J. R., pink with red blotch.

Watt, Mrs. wine red

White Belle, blush white

White Cluster, white with pink.

White Crepe, white with rose blotch

White Delight, flesh white.

White Giant, white

White Glory, white with iris blue

White Orchid, white with red throat

White Wonder, white

Whitney, D. J., geranium pink

Wilbrinck, light pink

Williamson, E. B., mallow purple

Wine Drop, wine.

Wistaria, orchid

Yellow Hammer, yellow

Yellow Treasure, yellow

Yellow Wonder, $(\mathrm{H})$ yellow

Zang, Gretchen, light pink

Zang, Tycko, pink.

Zenobia, orange red

One dozen for price of ten

in same variety and size

Large Medium Small

$11 / 4$ Up $3 / 4-11 / 4 \quad 1 / 4-3 / 4$

.75

.75

.20

.15

.10

.05

.10

.20

.10

.10

.15

.40

.75

.75

.85

.10

.05

.10

.10

2.00

.10

.10

1.00

.05

.20

.10

.15

.15

.75

1.50

.10

.10

.50

.25

.15

.10

.10

.10

.10

.10

.10

.15

.10

.10

.20

.05

.30

.10

.10

.05

.10

4.00

.05

.40

.10

.60

.15

.10

.07

$\cdots . . .7$

.15

1.25

.07

.30

.60

.60

5

.07

.07

.07

.07

.75

.15

.07

.10

.10

.60

.07

.07

.40

.20

.10

.07

.07

.07

.07

.07

.07

.10

.07

.07

.15

.20

.07

.07

.07

........

.30

.07

\section{.50}

.10

.07

.05

.05

.10

....................

.05

.20

.50

.50

.05

.05

.05

.05

.25

.10

.05

.07

.50

.05

.05

.30

.15

.07

.05

.05

.05

.05

.05

.05

.05

.05

.10

.10

.05

.05

.05

….....

.20

.05
Bulblets per

1,000

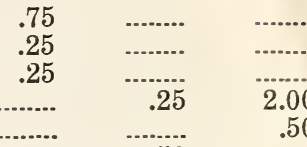

.50

.50

.50

.50

.75

.75

.75

........

.50

1.00

1.00

1.00

.50

.20

.20

.75

3.00

.20

.20

.50

.25

.20

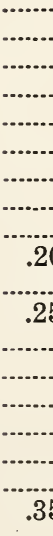




\section{BURBANK'S MIXTURE}

We have this mixture absolutely pure. Nothing but Burbank varieties in it. Purchased from him in 1926.

Prices: Large 50c per dozen; medium, 40c per dozen; small, 30c per dozen.

\section{KUNDERD'S GLORY MIXTURE}

Kept pure as purchased from Kunderd.

Prices: Same as Burbank's Mixture.

\section{BURBANK BULB COLLECTION}

$\begin{array}{ll}\text { Del Oro } & \text { Perla } \\ \text { Doro } & \text { Scarlet } \\ \text { Elena } & \text { Summit } \\ \text { Kiva } & \text { Tiger Face }\end{array}$

Above eight varieties originated by Luther Burbank January, 1926.

We will send you one large bulb each, the total for $\$ 5.00$.

\section{KUNDERD'S CHOICE BULBLET COLLECTION}

Purple Queen ....................................................... \$ .20

Dr. Nelson Shook ................................................. 15

Millionaire ….................................................... . .10

John T. McCutcheon ........................................ .15

J. D. Oliver …..................................................... . .20

Tiger ..................................................................... . 15

Ed. Springer ........................................................ .10

Lacinatus ................................................................... .

J. Ogden Armour ................................................ . .15

Evening Glory ....................................................... $\quad .10$

Blanche Bollinger .............................................. . 10

Cardinal Prince ...................................................... .15

Total

The Full Collection

$\$ 1.80$

$\$ 1.50$
KUNDERD'S IDEAL MIXTURE

Kept pure as purchased from Kunderd.

Prices: Same as Burbank's Mixture.

\section{LEGRONS' SPECIAL MIXTURE}

Prices by the dozen same as Burbank's Mixture. This mixture by the hundred, large $\$ 3.00$, medium $\$ 2.00$, small $\$ 1.00$. The full 100 must be taken to get this price.

\section{EUROPEAN BULBLET COLLECTION}

Heavenly Blue ..................................................\$1.00

Konynenburg, Mrs. Von........................................ $\quad .50$

LaGaiety ................................................................. .25

Veilchenblau .......................................................... .

Pfitzer's Triumph ............................................ $\quad .30$

Phaenemon …......................................................... .15

Caruso …….......................................................... .10

Herbstzauber ...................................................... .10

Orange Queen .................................................. . .10

Total European Collection for

$\$ 2.50$

\section{LEGRONS' SPECIAL COLLECTION}

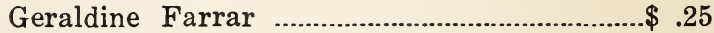

Gold Eagle ........................................................ .50

High Nye ............................................................ . .15

Blasco Ibanez ........................................................ .15

Mrs. P. W. Sisson................................................ .30

Rosemary ........................................................... . 10

Bernard Shaw ..................................................... 15

Victor ...................................................................

Joseph Field .................................................. .15

Geisha .................................................................

Total ............................................................ $\$ 2.05$

The Lot for ...................................................\$1.75
Chas V. Truax, Director,

Department of Agriculture
Richard Faxton, Chief, Division of Plant Industry

\section{State of Ohio \\ DEPARTMENT OF AGRICULTURE \\ Division of Plant Industry}

\section{CERTIFICATE OF NURSERY INSPECTION}

No. 915

Columbus, Ohio, October 11, 1927

THIS IS TO CERTIFY, That the nursery stock of

Le Gron Floral Company of Toledo, County of Lucas, State of Ohio, consisting of Gladioli, iris and greenhouse stock, has been inspected by a duly authorized inspector, in compliance with Section 1126, General Code, and has been found apparently free from dangerously injurious insects and plant diseases.

Permission is hereby granted the above mentioned nursery firm to sell and ship nursery stock which has been inspected for the year ending September 15, 1928.

This certificate, granted under the provisions of Section 1135, General Code, is invalid after September 15, 1928, and may be revoked earlier for cause.

N. B. All raspberry stock which may be covered by this certificate has been inspected twice, at least thirty days intervening between the two inspections.

Richard Faxon, Chief of Division

Chas. V. Truax, Director of Agriculture 
CULTURE. The culture of gladioli is quite simple. Ordinary fertile soil is all that is required. Sandy soil is best and clay is poorest. Cultivation and water are the main helps in growing good glads.

TIME OF PLANTING. Plant in spring as soon as frost is out of ground. The large bulbs will come in bloom first and the smaller sizes later, so the bloom of a single variety extends over quite a period. Then we have the early and late varieties which also prolong the blooming period. We also plant some bulbs about every two weeks up to July first and in this way have blooms to sell from July first until killed by frost.

SIZES. No. 1 is $1 \frac{1}{2}$ inch up; No. $2,1 \frac{1}{4}$ inch to

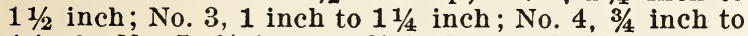
1 inch: No. $5,1 / 2$ inch to $3 / 4$ inch; No. $6,1 / 2$ inch and less. Bulblets are the small bulbs that form around the base of the larger bulbs. These always come true to name with the parent bulb.

PLANTING. We dig trenches six inches wide for all sizes. Nos. 1, 2 and 3 are planted four to six inches deep, putting two rows in a trench in zig-zag shape. These are placed by hand with right side of bulb placed up. Nos. 4,5 and 6 we sow in trenches about 2 to 3 inches deep regardless of position of bulbs. Bulblets are planted one inch deep and bottom of trench is covered with bulblets so that they entirely cover same.

The deep planting of large bulbs keeps wind from blowing them over after spikes have formed and also helps to hold moisture in dry seasons.

For field culture with the horse we have our rows 30 inches apart. This allows cultivator to cultivate the entire space by going once through. The rows must be straight. We always use lines in digging trenches.

CUTTING FLOWERS. Always cut flowers in morning when spikes are full of moisture. Spikes should be cut as soon as one or two flowers are open. Fill your vase with fresh water each day and cut one inch off the stems and you will have a nice bouquet for a week. The bottom flowers should be pulled off as they wilt. Always leave at least four leaves on plant in cutting. The main growth of the bulb and production of bulblets is after the spike is cut. Cultivation should not be neglected at this time.

DIGGING. We dig bulblets about September 1 . They can be dug when still quite green. We cut the tops off close to the ground with a sickle and then use a long handle round pointed shovel and shovel dirt and all into a sieve. The dirt is then rubbed or shook through the sieve. The large -bulbs are first loosened with a potato fork and then pulled up by hand and the tops broken off next to the bulb and the bulb and roots thrown into a tray six inches deep. Trays are then taken to cellar but windows are left open to give plenty of air.

STORAGE. After about two to three weeks from digging the roots and bulblets can be taken from the bulbs easily. After the bulbs are cleaned they can be put back in the trays but not over FOUR INCHES deep. Never store bulbs in same basement room with a furnace fire. If you have only up to a dozen bulbs of a kind you can use a 5-pound sugar sack nicely. Leave the top open. Temperature for winter should be between 35 and 40 degrees.
A $\$ 100.00$ Bulb For $\$ 5.00$

Read Mrs. Austin's. Letter Below.

GALLANT LEADER (Austin). A new LEADER among the Glads and one of Mrs. Austin's best productions. "In the field the majestic spikes, with many massive bloom open at one time, presents an almost startling effect of beauty. The opening buds are peach-red blending into geranium red in upper petals, while the lower ones show a blotch of white slightly dotted Begonia Rose deep in throat The spike is slender, straight, measures 64 inches in height, blooms 5 inches in diameter". Above measurements taken from stock grown on clay soil. Very prolific and a good seed bearer. Use this to impart vigor in your seedlings.

Each, $\$ 5.00$. No bulblets for sale. Only one bulb to a customer.

TROSSEAU PINK (Austin). Tall, graceful spike with well placed blooms of pleasing color difficult to describe. Tips touched Strawberry Pink blending to LaFrance Pink, deeper in throat with white blotch on lower petals, tinted maize yellow. A beautiful variety.

Each, \$2.00. No bulblets for sale.

RAVENNA CREAM (Austin). Tips of bloom are clear maize yellow shading almost to white in upper petals, while lower ones are overlaid buff yellow, beautifully flushed Tyrian pink. A lovely combination of color, and a bloom of good size, and open form.

Each, $\$ 1.50$. No bulblets for sale.

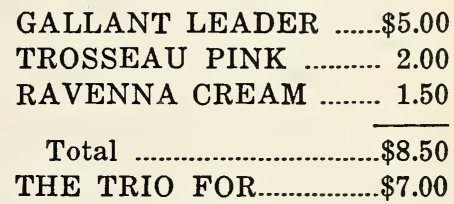

LeGron Floral Co.

Ravenna, Ohio, Dec. 4th, '26.

Dear Friends:

Toledo, Ohio.

I wish to commend you for the manner in which you are introducing the varieties purchased from us, in that you are pricing them reasonably according to the quality and quantity, thus placing them within the reach of the average grower.

Reasonable prices lowered gradually mean service and profit to your customers and to yourselves. GALLANT LEADER would compare favorably with those in the $\$ 100.00$ row but glad you are making it only $\$ 5.00$.

$$
\begin{aligned}
& \text { Yours very truly, } \\
& \text { MRS. A. H. AUSTIN. }
\end{aligned}
$$

We have purchased the entire stock of the above three varieties from the A. H. Austin Co. Send all orders to

LeGron Floral Co., R. 4, Toledo, Ohio. 
